\title{
Hydrocortisone treatment is associated with a longer duration of MODS in pediatric patients with severe sepsis and immunoparalysis
}

Katherine E. Bline ${ }^{1,2^{*}}$ D, Melissa Moore-Clingenpeel ${ }^{1,3}$, Josey Hensley ${ }^{1}$, Lisa Steele ${ }^{1}$, Kristin Greathouse ${ }^{1}$, Larissa Anglim', Lisa Hanson-Huber ${ }^{1}$, Jyotsna Nateri ${ }^{1}$, Jennifer A. Muszynski ${ }^{1,2}$, Octavio Ramilo ${ }^{1,4}$ and Mark W. Hall ${ }^{1,2}$

\begin{abstract}
Background: Severe critical illness-induced immune suppression, termed immunoparalysis, is associated with longer duration of organ dysfunction in septic children. mRNA studies have suggested differential benefit of hydrocortisone in septic children based on their immune phenotype, but this has not been shown using a functional readout of the immune response. This study represents a secondary analysis of a prospectively conducted immunophenotyping study of pediatric severe sepsis to test the hypothesis that hydrocortisone will be differentially associated with clinical outcomes in children with or without immunoparalysis.

Methods: Children with severe sepsis/septic shock underwent blood sampling within $48 \mathrm{~h}$ of sepsis onset. Immune function was measured by quantifying whole blood ex vivo LPS-induced TNFa production capacity, with a TNFa response $<200 \mathrm{pg} / \mathrm{ml}$ being diagnostic of immunoparalysis. The primary outcome measure was number of days in 14 with MODS. Univariate and multivariable negative binomial regression models were used to examine associations between hydrocortisone use, immune function, and duration of MODS.

Results: One hundred two children were enrolled (age 75 [6-160] months, 60\% male). Thirty-one subjects received hydrocortisone and were more likely to be older (106 [52-184] vs 38 [3-153] months, $p=0.04$ ), to have baseline immunocompromise (32 vs $8 \%, p=0.006)$, to have higher PRISM III (13 [8-18] vs 7 [5-13], $p=0.0003)$ and vasoactive inotrope scores (20 [10-35] vs 10 [3-15], $p=0.0002)$ scores, and to have more MODS days (3 [1-9] vs 1 $[0-3], p=0.002$ ). Thirty-three subjects had immunoparalysis (TNFa response 78 [52-141] vs 641 [418-1047] pg/ml, $p<0.0001)$. Hydrocortisone use was associated with longer duration of MODS in children with immunoparalysis after adjusting for covariables (aRR $3.7[1.8-7.9], p=0.0006$ ) whereas no association with MODS duration was seen in children without immunoparalysis (aRR $1.2[0.6-2.3], p=0.67$ ).

(Continued on next page)
\end{abstract}

\footnotetext{
* Correspondence: katherine.bline@nationwidechildrens.org

'The Abigail Wexner Research Institute at Nationwide Children's Hospital, Columbus, OH, USA

2Division of Critical Care Medicine, Nationwide Children's Hospital, 700 Children's Drive, Columbus, OH 43205, USA

Full list of author information is available at the end of the article
} 
(Continued from previous page)

Conclusion: Hydrocortisone use was independently associated with longer duration of MODS in septic children with immunoparalysis but not in those with more robust immune function. Prospective clinical trials using a priori immunophenotyping are needed to understand optimal hydrocortisone strategies in this population.

Keywords: Sepsis, Pediatric, Hydrocortisone, Shock, Immunoparalysis, MODS, Immune

\section{Background}

Septic shock is a leading cause of morbidity and mortality in children, with over 7000 pediatric deaths due to sepsis in the USA each year $[1,2]$. Septic shock is characterized by a dysregulated systemic immune response to infection that results in organ dysfunction. The failure of more than one organ, termed "multiple organ dysfunction syndrome (MODS)," confers a >10-fold increase in risk of mortality in critically ill children [3]. Current management guidelines for pediatric sepsis largely focus on supportive care, including fluid resuscitation and vasoactive medications, in addition to early empiric antibiotics [4]. Hydrocortisone is frequently prescribed as an adjunctive treatment for children with septic shock who have known adrenal insufficiency, those with a history of recent corticosteroid use, and those who remain hemodynamically unstable despite fluid resuscitation and initiation of vasoactive support $[5,6]$. The use of hydrocortisone in septic shock, however, remains controversial, with clinical trials in adults yielding conflicting results [7-9]. The current version of the pediatric Surviving Sepsis guidelines is unable to recommend for or against hydrocortisone use, largely due to a lack of evidence. The first prospective clinical trial of hydrocortisone use in pediatric septic shock is currently underway (NCT03401398).

The immune response to pediatric critical illness is highly dynamic, with acquired immune suppression frequently accompanying systemic inflammation. When severe, this compensatory immune suppression is termed "immunoparalysis." We and others have consistently shown that immunoparalysis is associated with adverse outcomes from critical illness in children including sepsis, trauma, and cardiopulmonary bypass [10-13]. We recently observed, in a prospective, single-center, 102subject cohort of children with severe sepsis/septic shock, that both immunoparalysis and hydrocortisone treatment were associated with longer duration of organ dysfunction. Prior transcriptomic work has suggested the possibility of a differential effect of hydrocortisone on pediatric sepsis outcomes depending on the host immune phenotype [14]. We therefore designed this secondary analysis of our data set to test the hypothesis that the relationships between hydrocortisone treatment and duration of MODS will be variable, depending on the presence or absence of immunoparalysis.

\section{Methods}

\section{Study population}

This is a secondary analysis of a prospective observational study which was conducted in the 54-bed medical-surgical pediatric intensive care unit (PICU) at Nationwide Children's Hospital, a quaternary-care children's hospital with over 3000 annual admissions. Children $<18$ years of age who were admitted to the PICU were eligible for enrollment if they met consensus criteria for severe sepsis or septic shock [15] within the preceding $48 \mathrm{~h}$. Subjects were excluded if a limitation-ofcare order was in place, if there was expected progression to brain death by the primary treating team, or if they were admitted to the cardiothoracic ICU. Written informed consent was obtained from subjects' legal guardians prior to participation, and if appropriate, subjects' assent was obtained. The protocol was approved by the Institutional Review Board at Nationwide Children's Hospital.

\section{Immune function}

Whole blood samples were collected within $48 \mathrm{~h}$ of sepsis onset. Complete blood count $(\mathrm{CBC})$ testing was done as part of routine care by the primary treating team, and all subjects had a CBC with differential completed within the first $48 \mathrm{~h}$ of sepsis onset. If multiple CBCs were obtained, the absolute cell counts reported are the lowest values within the first $48 \mathrm{~h}$. Plasma from un-stimulated blood samples was collected after centrifugation of whole blood at $1000 \times g$ for $5 \mathrm{~min}$ and stored at $-80^{\circ} \mathrm{C}$ to quantify interleukin (IL)-10 and IL-6, reflecting the systemic inflammatory response. Plasma TNF $\alpha$ levels were not measured, as previous studies have demonstrated that circulating TNF $\alpha$ comprises less than $10 \%$ of the measured ex vivo TNF $\alpha$ production capacity [16].

Innate immune function was measured by whole blood ex vivo lipopolysaccharide (LPS)-induced tumor necrosis factor (TNF)- $\alpha$ production capacity. Briefly, 50- $\mu$ l aliquots of heparinized whole blood were added to stimulation tubes containing LPS $(500 \mathrm{pg} / \mathrm{ml}$, phenol-extracted from Salmonella abortus equi [Sigma, St. Louis, MO]). Stimulation tubes were then incubated at $37^{\circ} \mathrm{C}$ for $4 \mathrm{~h}$. After incubation, supernatants were collected and stored at $-80^{\circ} \mathrm{C}$ for batched cytokine analysis. TNF $\alpha$ from LPS-stimulated supernatants were quantified by the Immulite 1000 automated chemiluminometer (Siemens 
Healthcare Diagnostics, Deerfield, IL). Immunoparalysis was defined as an LPS-induced TNF $\alpha$ production capacity (hereafter referred to as the TNF $\alpha$ response) $<200$ $\mathrm{pg} / \mathrm{ml}[17,18]$.

\section{Clinical data and outcome measurements}

For subjects with community-acquired sepsis, sepsis onset was defined as the initial time of presentation to any emergency department that resulted in hospitalization. For subjects who developed nosocomial sepsis, sepsis onset was defined as the time of transfer to the PICU. The electronic medical record was used to collect clinical data. Baseline immune compromise was defined as the presence of a congenital immunodeficiency, an oncologic diagnosis, or the receipt of chronic immunosuppressive therapy. Complex chronic conditions were defined as previously published [19]. The highest Pediatric Risk of Mortality (PRISM) III score within the first $24 \mathrm{~h}$ of sepsis onset was used to measure initial illness severity. The highest vasoactive inotrope score (VIS) in the first $48 \mathrm{~h}$, which is calculated using the combined doses of inotropic and vasopressor infusions [20], was used as a surrogate measure of subjects' shock state.

MODS was defined as dysfunction of more than one organ system according to previously published criteria [21] and represented only new organ dysfunction, not chronic organ dysfunction. The primary outcome measure was the number of days with MODS in the first 14 days from sepsis onset. Non-survivors were assigned the maximum number of MODS days (fourteen).

\section{Statistical analysis}

Values are reported as median and interquartile range (IQR) or $n$ and \% as appropriate. Simple comparisons were made using chi-square or Fisher's exact tests for categorical variables and Wilcoxon rank-sum tests for continuous variables. Univariate and multivariable negative binomial regression models were used to examine associations with duration of MODS. Coefficients for all negative binomial regression models were exponentiated to reflect risk ratios, or the percent increase in duration of MODS for a unit increase in the given predictor variable. Variable selection for the multivariable models was based on stepwise selection with an entry criterion of $p<0.15$ and an exit criterion of $p>0.1$, in addition to Akaike's Information Criterion (AIC, a measure of goodness of fit); VIS, baseline immune compromise, and hydrocortisone were retained in all models regardless of statistical significance on clinical grounds. To explore the possibility that an association between hydrocortisone use and outcomes may simply reflect the initial shock state of the subject, we performed secondary analysis limited to the subgroup of subjects with septic shock (those treated with vasoactive drugs). Statistical analyses were performed using SAS 9.4 (SAS Institute, Cary, NC) and Prism 7.0 (GraphPad Software, La Jolla, CA, USA).

\section{Results}

Subjects

A total of 102 subjects were enrolled between January 2012 and April 2014 with a median age of 75 (6-160) months. Subject demographic and clinical characteristics are shown in Table 1. Forty-eight patients had a complex chronic condition, most commonly including diagnoses of developmental delay or seizure disorder. There were 16 patients with baseline immune compromise due to receipt of solid organ or bone marrow transplant $(n=5)$, oncologic diagnosis $(n=6)$, congenital immunodeficiency $(n=2)$, or receipt of immunosuppressive medications for autoimmune disease $(n=3)$. Thirty-one subjects (30\%) received hydrocortisone. Of those, 14 (45\%) were known to have adrenal insufficiency, recent steroid use, or evidence of adrenal insufficiency with a cortisol level $<18 \mu \mathrm{g} / \mathrm{dl}$ in the setting of sepsis. Hydrocortisone-treated subjects were more likely to be older and to have baseline immune compromise, as well as a higher PRISM compared to those that did not receive hydrocortisone (Table 1). In the entire cohort, subjects that received hydrocortisone had a longer duration of MODS compared to those who did not receive hydrocortisone $(3[1-9]$ vs $1[1-3]$ days, $p=0.0002)$. In the subset with septic shock, this relationship was similar $(3.5[2-9]$ vs $2[0-3]$ days, $p<0.0001)$. All subjects who died were treated with hydrocortisone. There was no difference in the incidence of nosocomial infection between subjects treated with or without hydrocortisone in the cohort as a whole $(2 / 31[6 \%]$ vs $8 / 71[11 \%], p=0.72)$ or in the subgroup with septic shock $(1 / 26[4 \%]$ vs $5 / 54$ [9\%], $p=0.66$ ).

In multivariable analysis, the receipt of hydrocortisone in the first $48 \mathrm{~h}$, the presence of a complex chronic condition, and receipt of a red blood cell transfusion in the first $48 \mathrm{~h}$ were all independently associated with a higher adjusted relative risk (aRR) for longer duration of MODS in the complete cohort (Table 2). In the subset of subjects with septic shock, there was an even stronger independent association between hydrocortisone use and longer duration of MODS.

\section{Hydrocortisone and immune function}

All subjects underwent immune function testing during the first $48 \mathrm{~h}$ of sepsis, though $22 / 31$ (70\%) of the subjects in the hydrocortisone-treated group had blood sampling done after receiving at least one dose of hydrocortisone (median of $15.4 \mathrm{~h}$ ). In the cohort as a whole, subjects in the hydrocortisone-treated group had lower TNF $\alpha$ responses and lower absolute 
Table 1 Cohort characteristics

\begin{tabular}{|c|c|c|c|c|c|c|c|}
\hline \multirow[t]{2}{*}{ Characteristics } & \multicolumn{2}{|l|}{ All $(\boldsymbol{N}=102)$} & \multicolumn{2}{|c|}{ No hydrocortisone $(\boldsymbol{N}=71)$} & \multicolumn{2}{|c|}{ Hydrocortisone $(\boldsymbol{N}=31)$} & \multirow{2}{*}{$\begin{array}{l}p \\
\text { value }\end{array}$} \\
\hline & Median or $\boldsymbol{N}$ & IQR or $\%$ & Median or $\boldsymbol{N}$ & IQR or $\%$ & Median or $\boldsymbol{N}$ & IQR or $\%$ & \\
\hline Age, months & 74.5 & $(6,160)$ & 38 & $(3,153)$ & 106 & $(52,184)$ & 0.0441 \\
\hline Male & 61 & 60 & 40 & 56 & 21 & 68 & 0.2799 \\
\hline Complex chronic condition & 48 & 47 & 29 & 41 & 19 & 62 & 0.0571 \\
\hline Baseline immune compromise & 16 & 16 & 6 & 8 & 10 & 32 & 0.0057 \\
\hline RBC transfusion in first $48 \mathrm{~h}$ & 48 & 47 & 21 & 44 & 17 & 55 & 0.2983 \\
\hline Acute comorbidities & 18 & 18 & 14 & 20 & 4 & 13 & 0.4063 \\
\hline PRISM & 9 & $(5,14)$ & 7 & $(5,13)$ & 13 & $(8,18)$ & 0.0003 \\
\hline PELOD* & 7 & $(5,9)$ & 6 & $(5,9)$ & 8 & $(5,11)$ & 0.0813 \\
\hline VIS & 11.5 & $(5,20)$ & 10 & $(3,15)$ & 20 & $(10,35)$ & 0.0002 \\
\hline Mortality & 6 & 6 & 0 & 0 & 6 & 19 & 0.0005 \\
\hline \multicolumn{8}{|l|}{ Immune function: } \\
\hline Plasma IL6* (pg/mL) & 78.85 & $(22.7,258)$ & 63.9 & $(24,140)$ & 112 & $(17.9,811)$ & 0.2916 \\
\hline Plasma IL10* (pg/mL) & 30.55 & $(10,85.45)$ & 21.5 & $(10,75.9)$ & 35.9 & $(11.4,95.7)$ & 0.3586 \\
\hline Ex vivo TNFa" $(p g / m L)$ & 433 & $(143.5,821)$ & 465 & $(237.5,956.5)$ & 180.5 & $(52.3,633)$ & 0.0063 \\
\hline $\mathrm{AMC}^{\#}$ (cells/mm3) & 326.5 & $(93,648)$ & 320 & $(93,728)$ & 412 & $(75,545)$ & 0.6675 \\
\hline
\end{tabular}

RBC red blood cell, $P R I S M$ Pediatric Risk of Mortality Score, PELOD Pediatric Logistic Organ Dysfunction, AMC absolute monocyte count

*Highest value in first $48 \mathrm{~h}$

\#Lowest value in first $48 \mathrm{~h}$

lymphocyte counts compared to subjects not treated with hydrocortisone ( $p=0.0063$ and 0.0027 respectively) (Table 1). The degree of systemic inflammation, as evidenced by plasma IL- 6 and IL-10 levels, was similar between groups. Among subjects that received hydrocortisone, immune function and plasma cytokine levels were similar between subjects whose blood sample was obtained before versus after their first dose of hydrocortisone (TNF $\alpha$ response: 247 (65-408) vs $172(52-545) \mathrm{pg} / \mathrm{mL}, p=0.86$; IL-6: 167 (16-376) vs $70(18-3214) \mathrm{pg} / \mathrm{mL}, p=0.533$; IL-10: $36(15-76)$ vs $45(13-116) \mathrm{pg} / \mathrm{mL}, p=0.675)$. After adjusting for covariables, hydrocortisone treatment in the first $48 \mathrm{~h}$ of sepsis was independently associated with a longer duration of MODS in subjects with immunoparalysis $(\mathrm{aRR}=3.72$, 95\% CI 1.76-7.87, $p=0.0006)$ (Fig. 1a). This was not true for subjects without immunoparalysis $(\mathrm{aRR}=1.16,95 \% \mathrm{CI} 0.58-2.32, \mathrm{p}=0.67)$. This relationship was even stronger in the septic shock subgroup (Fig. 1b), with hydrocortisone use being associated with a longer duration of MODS only in subjects with immunoparalysis $(\mathrm{aRR}=4.57,96 \% \mathrm{CI}$ $2.31-9.07, p<0.0001$ vs $\mathrm{aRR}=1.54,95 \%$ CI 0.69 $3.43, p=0.29$ ).

\section{Discussion}

Ours is the first study to examine the relationships between the functional immune response, hydrocortisone use, and clinical outcomes in septic children. We were able to identify differential risk for prolonged organ dysfunction in children treated with hydrocortisone depending on their immune function in the acute phase of sepsis, with children with immunoparalysis demonstrating more prolonged MODS. Our data are in agreement with previously published mRNA studies [14] and highlight the need to incorporate prospective immunophenotyping into the design of future clinical trials of hydrocortisone in septic children.

Table 2 Adjusted risk of longer duration of MODS in entire cohort and those with septic shock

\begin{tabular}{|c|c|c|c|c|c|c|c|c|}
\hline \multirow[t]{2}{*}{ Variable } & \multicolumn{4}{|c|}{ Entire cohort } & \multicolumn{4}{|c|}{ Septic shock subset } \\
\hline & $\mathrm{aRR}$ & Lower CL & Upper CL & $p$-value & $\mathrm{aRR}$ & Lower CL & Upper CL & $p$ value \\
\hline Baseline immune compromise & 0.95 & 0.49 & 1.85 & 0.891 & 0.56 & 0.28 & 1.14 & 0.1117 \\
\hline $\mathrm{CCC}$ & 1.97 & 1.22 & 3.19 & 0.0054 & 1.73 & 1.09 & 2.76 & 0.0198 \\
\hline VIS & 1.01 & 1.00 & 1.03 & 0.0166 & 1.01 & 1.00 & 1.02 & 0.1236 \\
\hline RBC Transfusion & 1.83 & 1.18 & 2.83 & 0.0066 & 1.47 & 0.94 & 2.28 & 0.0882 \\
\hline Hydrocortisone & 1.79 & 1.06 & 3.04 & 0.0295 & 2.74 & 1.60 & 4.70 & 0.0002 \\
\hline
\end{tabular}

CCC complex chronic condition, VIS Vasoactive Inotrope Score, RBC red blood cell 

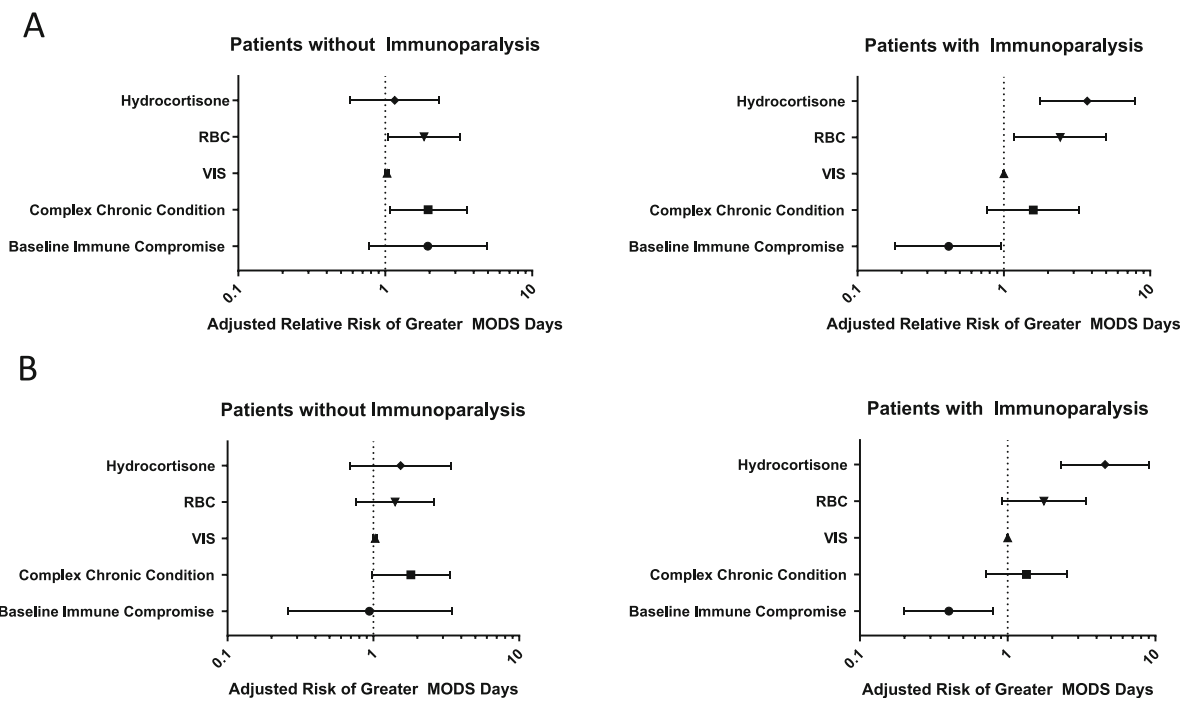

Fig. 1 Forest plot of the adjusted relative risk of variables on duration of MODS in the total cohort (a) and the subjects with septic shock (b). In both the total cohort and septic shock subset, subjects with immunoparalysis had a higher risk of a greater duration of MODS when receiving hydrocortisone treatment (aRR $3.72[1.76,7.87]$ and aRR $4.57[2.31,9.07]$, respectively)

Severe sepsis/septic shock remains a major source of morbidity and mortality worldwide. A recent multinational point prevalence study found a $25 \%$ mortality associated with pediatric severe sepsis [22] while another US multi-center study found a strong correlation between higher organ dysfunction scores and lower healthrelated quality of life in pediatric survivors of septic shock [23]. The mainstays of pediatric sepsis management include early fluid resuscitation, timely antibiotic administration, and hemodynamic support $[6,24,25]$ though hydrocortisone is frequently used as adjuvant therapy in children with fluid and catecholamineresistant shock as well as those with a priori risk factors for adrenal insufficiency. Hydrocortisone use in sepsis remains highly controversial, however. Evidence suggests that low-dose hydrocortisone treatment is associated with a shorter time to shock reversal in septic adults [26], but its reported effects on mortality have been inconsistent and at least one study has suggested an increase in nosocomial infection risk with hydrocortisone use [8]. Retrospective, observational pediatric data raise the possibility of equivocal or even harmful effects of hydrocortisone in the treatment of sepsis [27-29], but data from pediatric randomized controlled trials are, as yet, lacking. The current version of the pediatric Surviving Sepsis guidelines recommends neither for nor against the use of hydrocortisone [4].

While it is used in septic patients primarily for its hemodynamic-supporting mineralocorticoid activity, hydrocortisone does have some glucocorticoid activity which has the potential to exacerbate or perpetuate sepsis-induced immune suppression. The host immune response to sepsis is highly dynamic. The initial proinflammatory response is quickly accompanied by a compensatory downregulation of systemic immune function. This is termed "immunoparalysis" when severe and is characterized by a marked reduction of the ability of whole blood to produce TNF $\alpha$ upon ex vivo stimulation with LPS. We and others have repeatedly shown associations between reduction in the TNF $\alpha$ response and adverse outcomes including nosocomial infection, prolonged organ dysfunction, and death in critically ill children $[13,16,18]$. We recently published the results of a single-center, prospective immune phenotyping study of 102 children with severe sepsis/septic shock in which the use of hydrocortisone and severe reduction in the TNF $\alpha$ response were both associated with longer durations of MODS [17]. The degree to which hydrocortisone influences the host immune response in this setting is unknown. The current study represents a secondary analysis of this cohort, with emphasis on the relationships between hydrocortisone use, immunoparalysis, and MODS.

Our observation that hydrocortisone use was associated with a differential outcome depending on the subject's immunophenotype is complementary to a recent biomarker study conducted by Wong et al. Their group developed a panel of 5 plasma proteins (the PERSEVERE panel) that has been validated to risk-stratify children with acute septic shock [30]. They also developed a 100gene leukocyte transcriptomic panel that segregates children with acute septic shock into endotypes that are characterized by under-activation (endotype A) or overactivation (endotype B) of genes related to adaptive 
immunity and glucocorticoid receptor signaling [31]. In a secondary analysis of 288 children with acute septic shock, subjects in the intermediate and high-risk PERSEVERE groups who exhibited endotype B, hydrocortisone use was associated with a more than 10-fold reduction in in the risk of death or prolonged MODS. Hydrocortisone use was not associated with clinical outcomes in subjects with endotype A. This suggested that hydrocortisone benefit may be limited to children with a more activated immune state. Our study takes this line of reasoning further and, for the first time, provides evidence of an association between hydrocortisone use and worse outcomes in children who have severe functional innate immune impairment. It is therefore possible that prior clinical trials of hydrocortisone use in sepsis may have failed to correctly identify beneficial or harmful effects due to an inability to adjust for subjects' immunologic state. Since immunoparalysis is typically occult, and biomarkers of immunoparalysis are not currently measured in the clinical laboratory, it will be crucial to include prospective immune phenotyping in the design of future clinical trials of hydrocortisone in septic children.

Additionally, our results showed that receipt of a RBC transfusion was also associated with a higher risk of a longer duration of MODS, an observation that was recently the subject of another secondary analysis of this data set [32]. We also found that the presence of baseline immune compromise was associated with fewer MODS days in patients with immunoparalysis. In this patient population, immune suppression may be a modifiable risk factor through the tapering of immunosuppressive medication. Further, the presence of known baseline immune compromise may prompt earlier and more aggressive sepsis treatment. This is an area of active investigation.

This study was limited by its single center design and small sample size. Despite this, we were able to demonstrate significant associations between hydrocortisone use, immune function, and outcomes. The use of hydrocortisone in this study was not protocolized, and the resulting variability in prescription may have influenced our results. Perhaps most importantly, the majority of subjects who received hydrocortisone in our cohort underwent immune function testing after having received at least one dose of hydrocortisone. It is therefore unclear if hydrocortisone contributed to the development of immunoparalysis or if subjects at high risk for immunoparalysis are also more likely to receive hydrocortisone. The cause-and-effect relationship between hydrocortisone and innate immune suppression in critically ill children is an active area of investigation for our research group. The presence of a differential relationship between hydrocortisone use and outcomes depending on the host immune response, however, remains a key confounder of future clinical trials of hydrocortisone in pediatric sepsis. Lastly, this study focuses primarily on the TNF $\alpha$ response, which represents only one measure of immune function. While other aspects of immune function including phagocytosis, intracellular killing, antigen presentation, adaptive immune responses, and immunologic memory may be of interest in this population, a large body of literature suggests that the TNF $\alpha$ response is a highly clinically relevant readout of immune function in septic children.

\section{Conclusion}

The administration of hydrocortisone to children with immunoparalysis in the setting of severe sepsis/septic shock was associated with a longer duration of multipleorgan dysfunction syndrome whereas this association was not seen in children without immunoparalysis. While cause and effect relationships cannot be determined from this study, these data highlight the need to include prospective immunophenotyping in clinical trials of hydrocortisone in septic children.

\section{Acknowledgements \\ No acknowledgements.}

\section{Authors' contributions}

All authors participated in the study design and reviewed and approved the manuscript. JH, LS, KG, and LA participated in subject enrollment and clinical data collection. $\mathrm{LHH}$ and JN performed laboratory analyses. MMC performed statistical analyses. $\mathrm{KB}$ and $\mathrm{MH}$ created the initial draft of the manuscript.

\section{Funding}

There was no source of funding for the research related to this manuscript

Availability of data and materials

The datasets used and/or analyzed during the current study are available from the corresponding author on reasonable request.

Ethics approval and consent to participate

This study was approved by the Institutional Review Board at Nationwide Children's Hospital in Columbus, $\mathrm{OH}$.

\section{Consent for publication}

This manuscript does not contain any individual person's data in any form.

\section{Competing interests}

Dr. Mark Hall is a consultant for La Jolla Pharmaceuticals. This work is unrelated to the content of this manuscript.

\section{Author details}

${ }^{1} T$ The Abigail Wexner Research Institute at Nationwide Children's Hospital, Columbus, OH, USA. ${ }^{2}$ Division of Critical Care Medicine, Nationwide Children's Hospital, 700 Children's Drive, Columbus, OH 43205, USA ${ }^{3}$ Biostatistics Resource at Nationwide Children's Hospital, Columbus, OH, USA. ${ }^{4}$ Division of Infectious Diseases, Nationwide Children's Hospital, Columbus, $\mathrm{OH}$, USA. 
Received: 31 May 2020 Accepted: 26 August 2020

Published online: 04 September 2020

\section{References}

1. Watson RS, Carcillo JA, Linde-Zwirble WT, Clermont G, Lidicker J, Angus DC. The epidemiology of severe sepsis in children in the United States. Am J Respir Crit Care Med. 2003;167(5):695-701.

2. Hartman ME, Linde-Zwirble WT, Angus DC, Watson RS. Trends in the epidemiology of pediatric severe sepsis*. Pediatr Crit Care Med. 2013;14(7): 686-93.

3. Typpo K, Watson RS, Bennett TD, Farris RWD, Spaeder MC, Petersen NJ, et al. Outcomes of day 1 multiple organ dysfunction syndrome in the PICU. Pediatr Crit Care Med. 2019;20(10):914-22.

4. Weiss SL, Peters MJ, Alhazzani W, Agus MSD, Flori HR, Inwald DP, et al. Surviving Sepsis Campaign international guidelines for the management of septic shock and sepsis-associated organ dysfunction in children. Pediatr Crit Care Med. 2020;21(2):e52-e106.

5. Darmaros LF, Delgado AF, Carvalho WB. Corticosteroids in septic shock: what should the decision in pediatrics be? Rev Assoc Med Bras (1992). 2016; 62(6):482-4.

6. Davis AL, Carcillo JA, Aneja RK, Deymann AJ, Lin JC, Nguyen TC, et al. American College of Critical Care Medicine clinical practice parameters for hemodynamic support of pediatric and neonatal septic shock. Crit Care Med 2017:45(6):1061-1093.

7. Bollaert PE, Charpentier C, Levy B, Debouverie M, Audibert G, Larcan A Reversal of late septic shock with supraphysiologic doses of hydrocortisone. Crit Care Med. 1998;26(4):645-50.

8. Sprung CL, Annane D, Keh D, Moreno R, Singer M, Freivogel K, et al. Hydrocortisone therapy for patients with septic shock. N Engl J Med. 2008; 358(2):111-24.

9. Annane D, Briegel J, Keh D, Moreno R, Singer M, Sprung CL. Clinical equipoise remains for issues of adrenocorticotropic hormone administration, cortisol testing, and therapeutic use of hydrocortisone. Crit Care Med. 2003;31(8):2250-1 author reply 2-3.

10. Cornell TT, Sun L, Hall MW, Gurney JG, Ashbrook MJ, Ohye RG, et al. Clinical implications and molecular mechanisms of immunoparalysis after cardiopulmonary bypass. J Thorac Cardiovasc Surg. 2012;143(5):1160-6 e1.

11. Tschoeke SK, Ertel W. Immunoparalysis after multiple trauma. Injury. 2007; 38(12):1346-57.

12. Manzoli TF, Troster EJ, Ferranti JF, Sales MM. Prolonged suppression of monocytic human leukocyte antigen-DR expression correlates with mortality in pediatric septic patients in a pediatric tertiary intensive care unit. J Crit Care. 2016;33:84-9.

13. Muszynski JA, Nofziger R, Greathouse K, Nateri J, Hanson-Huber L, Steele L, et al. Innate immune function predicts the development of nosocomial infection in critically injured children. Shock. 2014;42(4):313-21.

14. Wong HR, Atkinson SJ, Cvijanovich NZ, Anas N, Allen GL, Thomas NJ, et al. Combining prognostic and predictive enrichment strategies to identify children with septic shock responsive to corticosteroids. Crit Care Med. 2016:44(10):e1000-3.

15. Goldstein B, Giroir B, Randolph A. International pediatric sepsis consensus conference: definitions for sepsis and organ dysfunction in pediatrics. Pediatr Crit Care Med. 2005;6(1):2-8.

16. Hall MW, Geyer SM, Guo CY, Panoskaltsis-Mortari A, Jouvet P, Ferdinands J, et al. Innate immune function and mortality in critically ill children with influenza: a multicenter study. Crit Care Med. 2013:41(1):224-36.

17. Muszynski JA, Nofziger R, Moore-Clingenpeel M, Greathouse K, Anglim L, Steele $L$, et al. Early immune function and duration of organ dysfunction in critically III children with sepsis. Am J Respir Crit Care Med. 2018;198(3):361-9.

18. Hall MW, Knatz NL, Vetterly C, Tomarello S, Wewers MD, Volk HD, et al. Immunoparalysis and nosocomial infection in children with multiple organ dysfunction syndrome. Intensive Care Med. 2011;37(3):525-32.

19. Feudtner C, Christakis DA, Connell FA. Pediatric deaths attributable to complex chronic conditions: a population-based study of Washington State, 1980-1997. Pediatrics. 2000;106(1 Pt 2):205-9.

20. Gaies MG, Gurney JG, Yen AH, Napoli ML, Gajarski RJ, Ohye RG, et al. Vasoactive-inotropic score as a predictor of morbidity and mortality in infants after cardiopulmonary bypass. Pediatr Crit Care Med. 2010;11(2):234-8.

21. Villeneuve A, Joyal JS, Proulx F, Ducruet T, Poitras N, Lacroix J. Multiple organ dysfunction syndrome in critically ill children: clinical value of two lists of diagnostic criteria. Ann Intensive Care. 2016;6(1):40.
22. Weiss SL, Fitzgerald JC, Pappachan J, Wheeler D, Jaramillo-Bustamante JC, Salloo A, et al. Global epidemiology of pediatric severe sepsis: the sepsis prevalence, outcomes, and therapies study. Am J Respir Crit Care Med. 2015;191(10):1147-57.

23. Zimmerman JJ, Banks R, Berg RA, Zuppa A, Newth CJ, Wessel D, et al. Trajectory of mortality and health-related quality of life morbidity following community-acquired pediatric septic shock. Crit Care Med. 2020;48(3):329-37.

24. Sterling SA, Miller WR, Pryor J, Puskarich MA, Jones AE. The impact of timing of antibiotics on outcomes in severe sepsis and septic shock: a systematic review and meta-analysis. Crit Care Med. 2015;43(9):1907-15.

25. de Oliveira CF, de Oliveira DS, Gottschald AF, Moura JD, Costa GA, Ventura AC, et al. ACCM/PALS haemodynamic support guidelines for paediatric septic shock: an outcomes comparison with and without monitoring central venous oxygen saturation. Intensive Care Med. 2008;34(6):1065-75.

26. Annane D, Bellissant E, Bollaert PE, Briegel J, Keh D, Kupfer Y. Corticosteroids for severe sepsis and septic shock: a systematic review and meta-analysis. BMJ. 2004;329(7464):480.

27. Nichols B, Kubis S, Hewlett J, Yehya N, Srinivasan V. Hydrocortisone therapy in catecholamine-resistant pediatric septic shock: a pragmatic analysis of clinician practice and association with outcomes. Pediatr Crit Care Med. 2017;18(9):e406-e14

28. Markovitz BP, Goodman DM, Watson RS, Bertoch D, Zimmerman J. A retrospective cohort study of prognostic factors associated with outcome in pediatric severe sepsis: what is the role of steroids? Pediatr Crit Care Med. 2005;6(3):270-4.

29. Zimmerman JJ, Donaldson A, Barker RM, Meert KL, Harrison R, Carcillo JA, et al. Real-time free cortisol quantification among critically ill children. Pediatr Crit Care Med. 2011;12(5):525-31.

30. Wong HR, Weiss SL, Giuliano JS Jr, Wainwright MS, Cvijanovich NZ, Thomas $\mathrm{NJ}$, et al. The temporal version of the pediatric sepsis biomarker risk model. PLoS One. 2014;9(3):e92121.

31. Wong HR, Cvijanovich NZ, Anas N, Allen GL, Thomas NJ, Bigham MT, et al. Developing a clinically feasible personalized medicine approach to pediatric septic shock. Am J Respir Crit Care Med. 2015;191(3):309-15.

32. Srouji LS, Moore-Clingenpeel M, Hensley J, Steele L, Greathouse K, Anglim L, et al. Shock severity modifies associations between RBC transfusion in the first 48 hours of sepsis onset and the duration of organ dysfunction in critically ill septic children. Pediatr Crit Care Med. 2020;21(8):e475-e84.

\section{Publisher's Note}

Springer Nature remains neutral with regard to jurisdictional claims in published maps and institutional affiliations.
Ready to submit your research? Choose BMC and benefit from:

- fast, convenient online submission

- thorough peer review by experienced researchers in your field

- rapid publication on acceptance

- support for research data, including large and complex data types

- gold Open Access which fosters wider collaboration and increased citations

- maximum visibility for your research: over $100 \mathrm{M}$ website views per year

At BMC, research is always in progress.

Learn more biomedcentral.com/submissions 